\title{
QRS detection using S-Transform and Shannon Energy
}

\author{
Z.Zidelmal,a, , A.Amirou, ${ }^{\text {,a, }}$, D.Ould-Abdeslam,b, A.Moukadem,b, \\ A.Dieterlen, ${ }^{\text {, }}$ \\ ${ }^{a}$ Mouloud Mammeri University, Tizi-Ouzou, Algeria \\ ${ }^{b}$ MIPS Laboratory, University of Haute Alsace, 68093, Mulhouse Cedex, France
}

\begin{abstract}
This paper presents a novel method for QRS detection in electrocardiograms (ECG). It is based on the S-Transform, a new time frequency representation (TFR). The S-Transform provides frequency-dependent resolution while maintaining a direct relationship with the Fourier spectrum. We exploit the advantages of the S-Transform to isolate the QRS complexes in the timefrequency domain. Shannon energy of each obtained local spectrum is then computed in order to localize the $\mathrm{R}$ waves in the time domain.

Significant performance enhancement is confirmed when the proposed approach is tested with the MIT-BIH arrhythmia database (MITDB). The obtained results show a sensitivity of $99.84 \%$, a positive predictivity of $99.91 \%$ and an error rate of $0.25 \%$. Furthermore, to be more convincing, the authors illustrated the detection parameters in the case of certain ECG segments with complicated patterns.
\end{abstract}

Key words: QRS detection, S-Transform, Shannon Energy.

Email addresses: z-zidelmal@mail.ummto.dz (Z.Zidelmal ), a-amirou@mail.ummto.dz (A.Amirou ), djaffar.ould-abdeslam@uha.fr (D.Ould-Abdeslam ), ali.moukadem@uha.fr (A.Moukadem ), alain.dieterlen@uha.fr (A.Dieterlen ) 


\section{Introduction}

Electrocardiography is an important tool in diagnosing the condition of the heart. The Electrocardiogram (ECG) is a signal that records the electrical activity of the heart. It is characterized by its main waves: P, QRS and T. QRS complex is the most striking waveform within the ECG. It represents the depolarization phenomenon of the ventricles and yields useful information about their behavior.

Since the QRS complex is the dominant feature of the ECG signal, its detection is a crucial part of any ECG analysis algorithm. Once the positions of the QRS complexes are found, the locations of other components like P, $\mathrm{T}$ waves and ST segment, etc. are found relative to the position of the QRS, in order to analyze the complete cardiac period. QRS detection can be done rather easily by the trained eye of cardiologist. Unluckily, the problem of automation of this process is difficult, not only because of the physiological variability of the QRS complexes, but also because of the various types of noise that can be present in the ECG signal. Noise sources include muscle noise, artifacts due to electrode motion, power-line interference, baseline wander, $\mathrm{P}$ and $\mathrm{T}$ waves with high-frequency characteristics similar to QRS complexes.

Within the last decades, a wide diversity of QRS detection algorithms have been proposed in the literature. In [1], B.U.Köhler et al. have summarized and compared the most important approaches proposed until 2000. References $[2,3,4]$ represent original works on this subject. Such algorithms are based on digital filters and ECG signal derivative. This points out the usage of the characteristic steep slop of the QRS for its detection. Other 
algorithms are based on more sophisticated filters [5, 6, 7], filters and Hilbert transform [8]. Some algorithms operate in the time domain. $\mathrm{R}$ peak detection based on zero crossing counts is proposed in [9]. In [10], I.Christov has used adaptive thresholds considering noise level and low amplitude beats. Wavelet transform has also been widely used for QRS detection [11, 12, 13, 14, 15, 16] but along with its flexibility, comes the price of determining the appropriate mother wavelet and level decomposition. Other methods were based on more complex approaches, neural network [12, 17], Support Vector Machines [18], genetic algorithms [19], Hidden Markov Models [20], K-Nearest Neighbor [21] and combination of different algorithms working in parallel [22, 23].

Although the performance of these algorithms, high detection accuracy still remains open problems. The currently achievable detection rates hide the problems that are still present in case of very noisy or pathological signals. A satisfying solution to these problems is still not found.

QRS detection algorithm in this paper is based on the S-Transform (ST). After ECG time-frequency decomposition, Shannon energy of local spectra is used to localize each QRS complex. The proposed algorithm was validated against the standard MIT-BIH arrhythmia database (MITDB).

The paper is organized as follows. After an introductory, section 2 presents the S-Transform principle and how the QRS complexes are localized in the time domain. Accordingly, an algorithm of $\mathrm{R}$ peak detection is developed. The proposed method is tested in Section 3. Finally, Section 4 concludes the paper. 


\section{Method}

\subsection{MIT-BIH arrhythmia database}

To analyze QRS detector performance, it is necessary to use a standard database, so obtained results can be interpreted and compared to other results. Several standard ECG databases are available for the evaluation of software QRS detection algorithms. Tests on these annotated databases provide reproducible and comparable results. Furthermore, these databases contain atypical waveforms, considerable artifacts and broad variety of QRS morphologies.

Frequently, the annotated MIT-BIH Arrhythmia Database [25] is used. This database contains 48 records. Each record is of 30 minutes length with a sampling frequency $f_{s}=360 \mathrm{~Hz}$. The data were recorded in two channels (modified limb lead II and one of the modified leads V1, V2, V4 or V5) of surface ECG [25]. Since normal QRS complexes might be usually prominent in lead II, this lead was used as a default. However, some segments with saturation are replaced by those of the other lead (eg, record 116: 1354.2s1361.9s). Two cardiologists have annotated all beats. Approximately $70 \%$ of the beats are annotated as Normal.

The complete database was considered, except for record 207, where a data segment showing ventricular flutter waves, for a total of $142.5 \mathrm{~s}$ are excluded. Note that these intervals are not annotated.

\subsection{S-Transform principal}

The S-Transform was proposed in 1996 by R.G.Stockwell [24]. It provides frequency-dependent resolution while maintaining a direct relationship with 
the Fourier spectrum. The ST originates from two advanced signal processing tools, the short time Fourier transform (STFT) and the wavelet transform (WT). It can be viewed as a frequency dependant STFT or a phase corrected wavelet transform. This transform provides a good time and frequency resolution. In addition, it allows us access to any frequency component in the time-frequency domain without requiring to any digital filter.

Derived from the STFT, the standard ST of a time varying signal $x(t)$ is given by:

$$
S(\tau, f)=\int_{-\infty}^{+\infty} x(t) w(t-\tau) e^{-i 2 \pi f t} d t
$$

where $w(t)$ is a time window centered in $t=0$ and used to extract a segment of $x(t)$. The S-Transform can be found by defining a particular window function $w(t)$, a normalized gaussian.

$$
w(t)=\frac{1}{\sigma \sqrt{2 \pi}} e^{\frac{-t^{2}}{2 \sigma^{2}}}
$$

where $\sigma$ characterizes the width of the gaussian window. Note that the gaussian window was chosen since it is the most compact in time and frequency. We allow $w(t)$ a translation $\tau$ and a dilatation (a variable width $\sigma$ ). A constraint is added to restrict the window width $\sigma$ to be a function of the frequency

$$
\sigma(f)=\frac{1}{|f|}
$$

so equation 2 becomes

$$
w(t-\tau)=\frac{|f|}{\sqrt{2 \pi}} e^{\frac{-(t-\tau)^{2} f^{2}}{2}}
$$

The function S-Transform is then rephrased as

$$
S(\tau, f)=\frac{|f|}{\sqrt{2 \pi}} \int_{-\infty}^{+\infty} x(t) e^{\frac{-(t-\tau)^{2} f^{2}}{2}} e^{-i 2 \pi f t} d t
$$


where $\tau$ and $f$ denote respectively the time of the spectral localization and Fourier frequency. The window width $\sigma$ varying inversely with frequency makes S-Transform performing a multi-resolution analysis on the signal. At each instant $t_{o}$, the one dimensional function of the frequency variable $f$ defined by $S\left(t_{o}, f\right)$ is a local spectrum. The one dimensional function of the time variable $\tau$ and a fixed frequency $f_{o}$ defined by $S\left(\tau, f_{o}\right)$ is called a voice.

In equation (5), the S-transform can be seen as a continuous wavelet transform $W(\tau, d)$ with a specific mother wavelet and a phase factor correction $[24]$

$$
S(\tau, f)=W(\tau, d) e^{-i 2 \pi f \tau}
$$

with a constraint that the wavelet dilatation factor $d=\sigma(f)$. hence, the mother wavelet is

$$
\psi(t, f)=\frac{|f|}{\sqrt{2 \pi}} e^{\frac{-t^{2} f^{2}}{2}} e^{-i 2 \pi f t}
$$

Note that the wavelet (7) does not satisfy the admissibility condition of having a zero mean, so (6) is not strictly a CWT. The ST returns correct amplitude response for all frequencies and produces a time frequency representation instead of the time scale representation developed by the CWT. These two representations were compared in [26].

For a time series $x(k T)$ corresponding to $x(t)$ with $k=0, . ., N-1$ and $T$, the time sampling interval, the discrete Fourier transform (DFT) is

$$
X\left(\frac{n}{N T}\right)=\frac{1}{N} \sum_{k=0}^{N-1} x(k T) e^{\frac{-i 2 \pi n k}{N}}
$$

where $n=0, . ., M-1$ and $M$, the length of the DFT. The discrete version of S-Transform is given by:

$$
S(j, n)=\sum_{m=0}^{N-1} X(m+n) W(m, n) e^{\frac{i 2 \pi m j}{N}} ; \quad n \neq 0
$$


where $W(m, n)=e^{\frac{-2 \pi^{2} m^{2}}{n^{2}}}, X($.$) are obtained by shifting the DFT of x(k T)$ and $\left(\frac{n}{N T} \rightarrow f, j T \rightarrow \tau\right)$. For $n=0,(f=0)$, we have the average of the time series at the zero frequency voice $[24,26]$ :

$$
S(j, 0)=\frac{1}{N} \sum_{m=0}^{N-1} x\left(\frac{m}{N T}\right)
$$

The output of the discrete S-Transform is an N by M matrix called STmatrix where rows represent the time and columns, the frequencies. In this paper, a frequency range $\left[0 H z-\frac{f_{s}}{2} H z\right]$ is used to cover the composite ECG signal.

\subsection{QRS localization}

To study the QRS frequency content, four heartbeat types are considered: Normal beats (N), Premature Ventricular Contractions (PVC), Left and Right Bundle Branch Blocks (L and R). In a previous work [15], we showed that the frequency content of these beats representing the predominant classes of the considered database belongs essentially to the frequency band $[5 \mathrm{~Hz}-22.5 \mathrm{~Hz}]$. This can be also seen in Fig.1. For this illustration, we have selected an ECG segment from record 119 containing normal beats, $\mathrm{N}$ (shifting to high frequencies) and PVC beats (shifting to low frequencies). In this figure, one can see that if we consider the frequency band cited above, the effect of $\mathrm{P}$ and $\mathrm{T}$ waves will be removed.

\subsubsection{Shannon energy}

In order to detect QRS complexes in the time-frequency plane, we worked within the range of frequencies $[5 \mathrm{~Hz}-22.5 \mathrm{~Hz}]$, where the heartbeats are more pertinent. Accordingly, we exploit the already proposed method in [27] to 

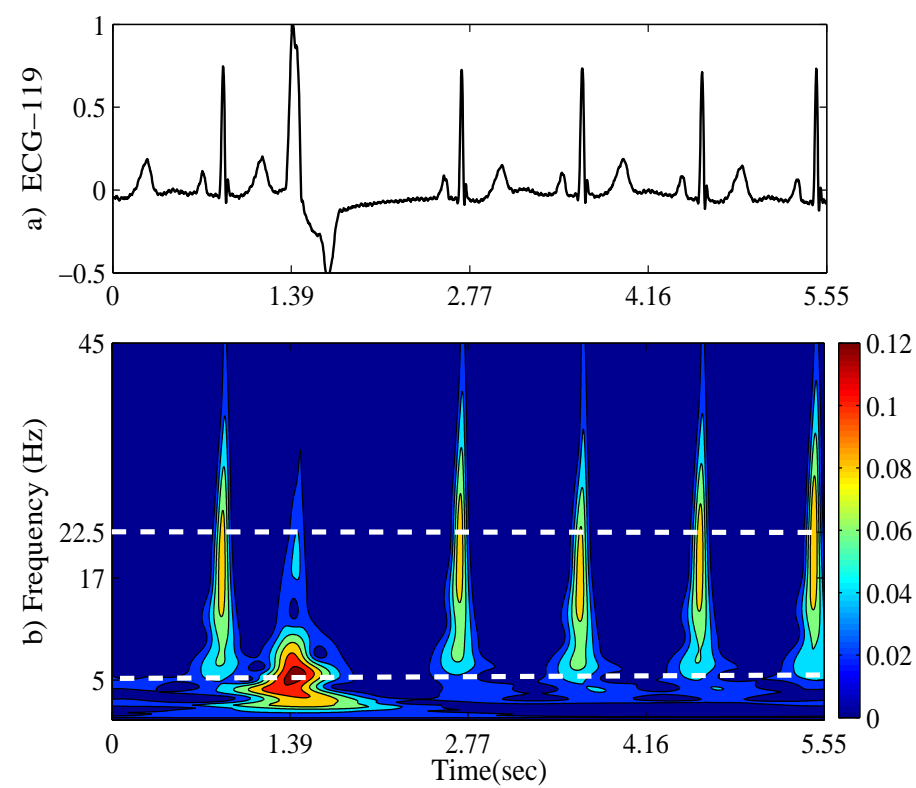

Figure 1: ECG signal (panel a) and ST-Spectrogram of the signal (panel b). The ST returns correct amplitude response for all frequencies. This amplitude means the same as the amplitude of Fourier transform. The color bar scale shows the relationship between spectrogram amplitude and color. For better illustration, the frequency axis was limited to $45 \mathrm{~Hz}$.

heart sounds segmentation using Shannon energy of local spectra. Shannon energy (SSE) calculates the energy of the local spectrum obtained with ST for each sample $(j)$ of the original ECG. Therefore, the SSE of each row " $j$ " of the extracted ST-matrix in the selected frequency band is:

$$
S S E(j)=-\sum_{n=n_{o}}^{n_{1}}[S(j, n)]^{2} \log [S(j, n)]^{2}
$$


where $n_{o}$ and $n_{1}$ correspond respectively to $f_{o}=5 \mathrm{~Hz}$ and $f_{1}=22.5 \mathrm{~Hz}$. The normalized Shannon energy is expressed by:

$$
S S E_{n}=\frac{S S E}{\mid \max (S S E \mid}
$$

The advantage of using Shannon energy instead of squared energy is its capacity to emphasis medium and low QRSs better than the classical squared energy (see Fig.2). This feature is useful in the case of significant QRS ampli-

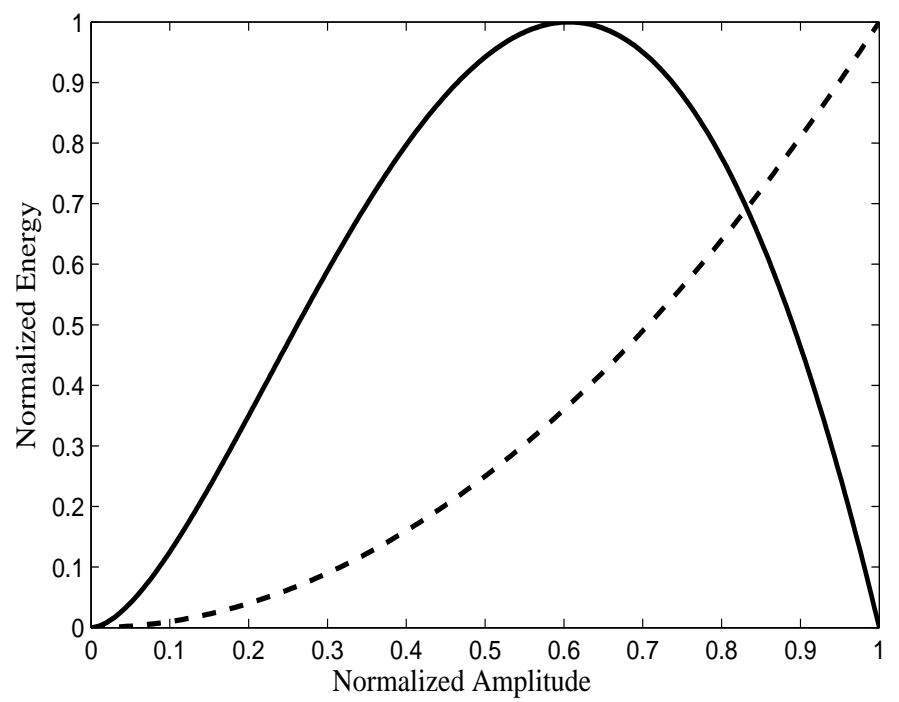

Figure 2: Comparison of normalized energy values obtained with different methods, Shannon energy (solid) and squared energy (dashed).

tude changes. Fig.3.c illustrates the normalized Shannon energy computed using a segment of record MIDB:107. The $S S E_{n}$ signal highlights the QRS complexes and reduces the other components.

It should be noted that QRS location can be done using various methods such as multiresolution analysis [15], filter banks [5] and mainly bandpass 

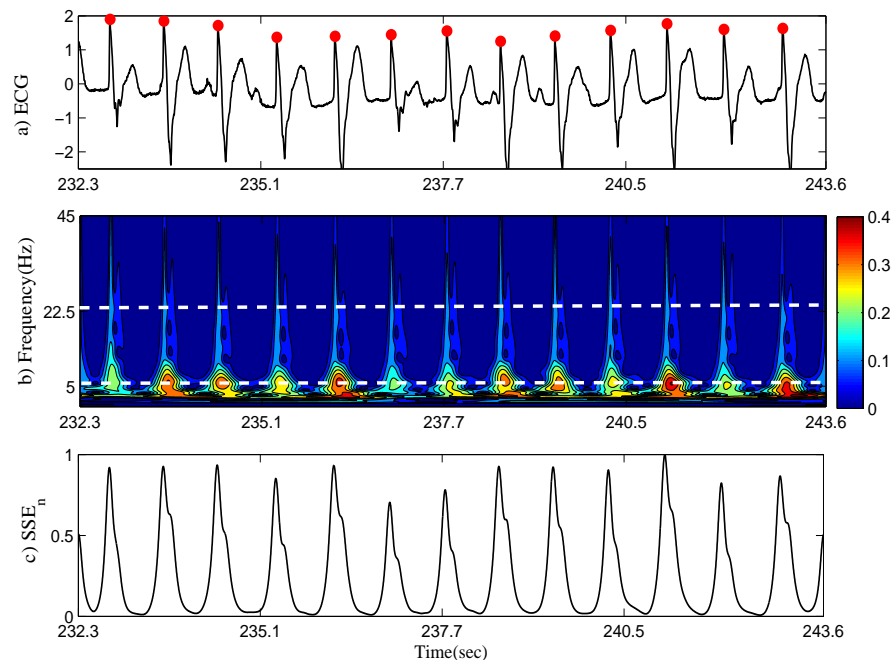

Figure 3: a) ECG waveform, segment of record 107 with paced beats; b) ST spectrogram of the ECG. The color bar scale shows the relationship between spectrogram amplitude and color; c) Local Shannon energy obtained with the selected frequency band.

filtering $[1,2,3,6]$. Fig.4 shows a brief comparison between the proposed approach and bandpass filtering. The bandpass filtered ECG presents distortions due to the calculation process. This needs additional signal processing steps. In addition, the maxima are slightly shifted from the $\mathrm{R}$ peak positions. However, Shannon energy exhibits a smooth envelope where the effect of $\mathrm{P}$ and $\mathrm{T}$ waves is completely removed. Its maxima have the same positions as the $\mathrm{R}$ peaks. This combination of desirable features motivated us to retain the SSE signal as a temporal detection parameter.

The following algorithm summarizes the procedure used for QRS detection using Shannon envelope computed on the extracted ST matrix. 


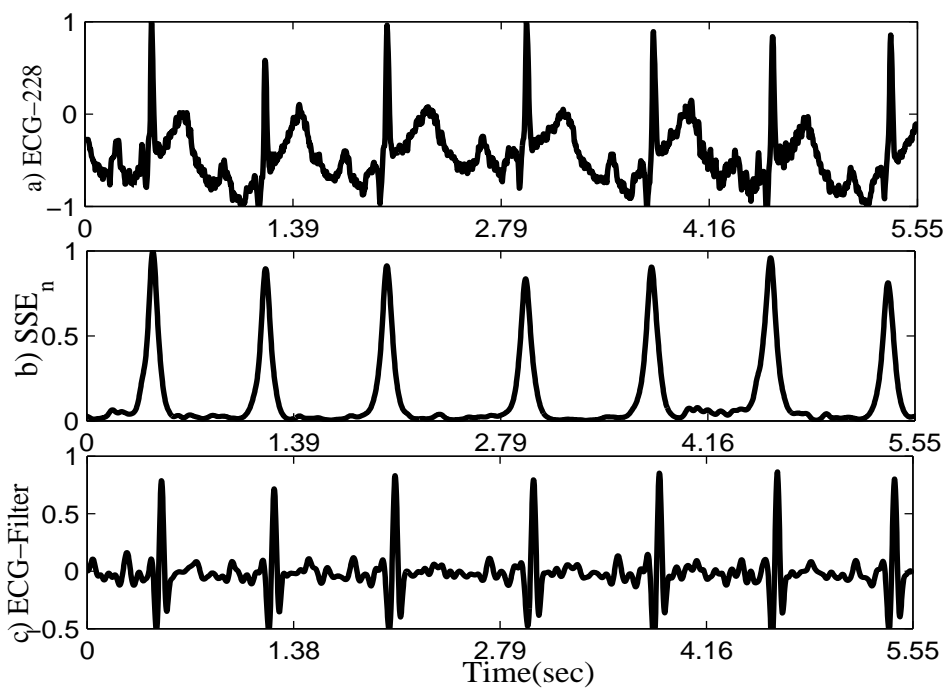

Figure 4: a) Plot of ECG waveform, segment of record 228; b) Local Shannon energy obtained with the selected frequency band; c)bandpass filtered ECG.

\subsubsection{Algorithm}

1. Apply S-transform to ECG signal $x(j)$,

2. Compute the normalized Shannon energy $S S E_{n}$ on the selected frequency band,

3. QRS localization: Select $S S E_{n}(j)$ associated to QRS complex using a threshold $\lambda=0,3 * \max \left(S S E_{n}\right)$,

- if $S S E_{n}(j) \geq \lambda$ then position $j \in Q R S$ (candidate),

- else position $j \not \mathrm{QRS}$,

4. Identify different QRS complexes: if $j, j^{\prime}$ are consecutive selected positions: $\left|j-j^{\prime}\right|<36$ then $\left(x(j), x\left(j^{\prime}\right)\right) \in$ same QRS else $\left(x(j), x\left(j^{\prime}\right)\right) \notin$ same QRS . $\left(36 * f_{s}=100 m s\right.$ is the standard QRS duration). 
5. Elimination of multiple detection: a peak occurring within the refractory period (200 ms) is disregarded. This constraint is a physiological one due to the refractory period during which ventricular depolarization cannot occur (QRS cannot occur).

6. Searchback for eventual missed QRS complexes: If no QRS complex is detected within $150 \%$ of the current $\mathrm{RR}$ interval, then we apply a searchback using a secondary threshold of 0.5 times the previous one to find any missed peaks $[3,4,15]$.

\section{Evaluation results and discussions}

In order to evaluate the performance of our algorithm, all considered ECG records was analyzed.

The principle of our algorithm is the access to any voice, hence, to the frequency content of the signal of interest (QRS complex) without recourse to filtering to remove the effect of noise, $\mathrm{P}$ and $\mathrm{T}$ waves. As a first application, we examine the localization of QRS complexes by avoiding the effect of $\mathrm{P}$ and $\mathrm{T}$ waves. For this, we used a segment of record 201 containing PVC beats and tall peaked $\mathrm{T}$ waves. Fig.5.a plots the original ECG waveforms corresponding to the ranges of $[638.8 s-650.3 s]$. The ST spectrogram displayed in Fig.5.b shows that in the selected frequency band $[5 \mathrm{~Hz}-22.5 \mathrm{~Hz}]$, the energy corresponds essentially to the QRS complexes. We note that the $\mathrm{P}$ and $\mathrm{T}$ waves belong to the frequency band $[0-5 H z]$. This allows us to isolate these QRSs in the time-frequency domain. In Fig.5.c, one can see that Shannon envelope leads to a correct enhancement of all R-peaks.

To show the robustness of the proposed method, we found it necessary 


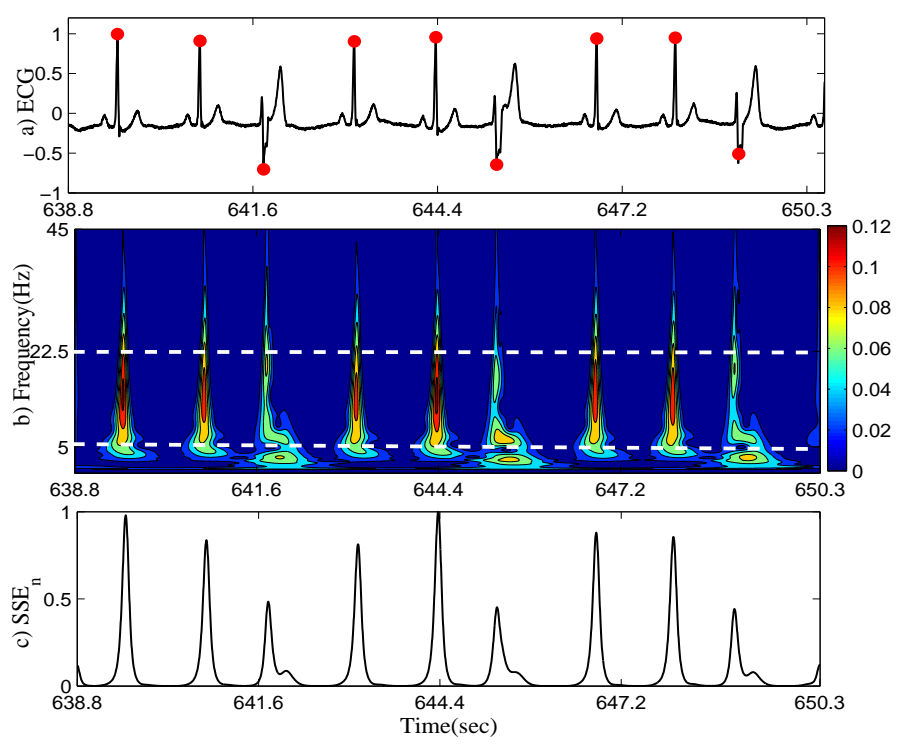

Figure 5: a) Plot of ECG waveform from record 201: [638.8s - 650.3]s with ventricular tachycardia; b) ST spectrogram of the ECG, the color bar scale shows the relationship between spectrogram amplitude and color; c) Shannon energy of the selected frequency band. The bullets represent the position of detected $\mathrm{R}$ peaks.

to illustrate the QRS detection parameters on some selected ECG segments from records with abnormal beats and severe artifacts such as records (104, 105, 108, 203 and 228). These records are those for which most algorithms found in literature obtained worst results $[1,2,5,9,15]$.

Tape 104, original ECG of a 66 years old woman is characterized by occurrence of several bursts of muscle noise. In Fig.6.b, we can see that the muscle artifacts are shifted to high frequencies. This record is surely a good case for showing how the effect of high frequency noises is removed without filtering. We obtained a perfect extraction of the QRSs in the time-frequency domain, so Shannon energy localizes all the corresponding $\mathrm{R}$ peaks in the 
time domain. The artifacts amplitude in the segment $[318 s-321 s]$ is higher than that of the $\mathrm{R}$ waves but significantly attenuated in the localization signal $S S E_{n}$. Hence, all R peaks are accurately detected (Fig.6.a).
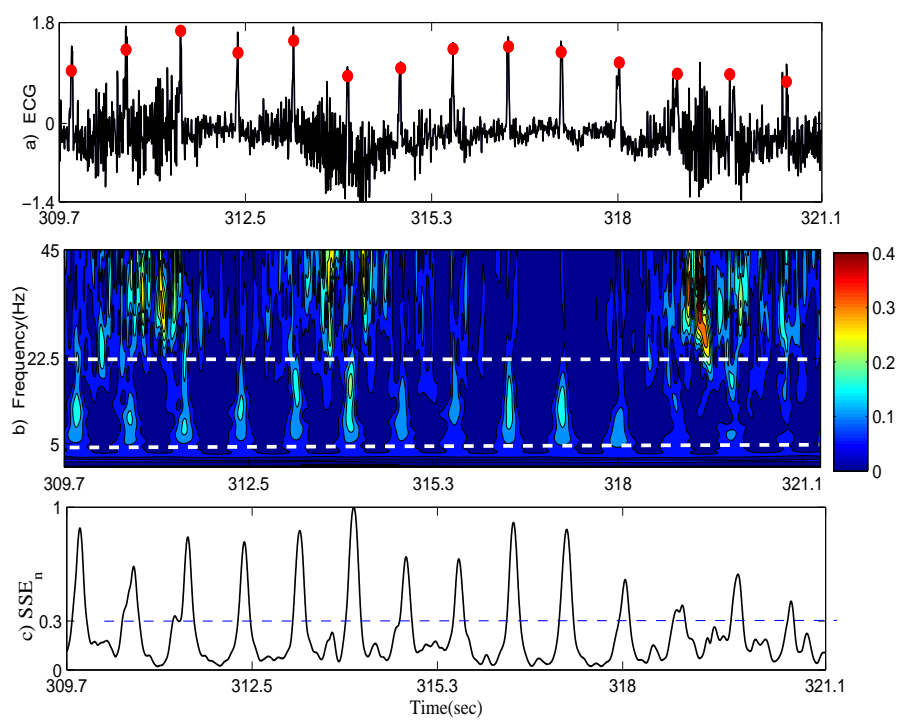

Figure 6: a) Plot of ECG waveform from record 104: 309.7s - 321.1s with muscle noise; b) ST spectrogram of the ECG, the color bar scale shows the relationship between spectrogram amplitude and color; c) Shannon energy of the selected frequency band. The bullets represent the position of detected $\mathrm{R}$ peaks.

Record 105 is an ECG of a 73 years old woman. The predominant feature of this tape is high-grade noise, artifacts, abnormal shapes and unclassifiable beats. especially at the episodes $[1223 s-1247 s],[1322 s-1333 s],[1647 s-$ $1661 s]$ and $[1688 s-1697.2 s]$. Fig. 7.a plots the original ECG waveforms corresponding to the episode $[1223 s-1237 s]$. It seems difficult, even for human eye to discriminate clearly the ECG beats from the cases with serious distortion of the ECG. There is a broad variety of QRS morphologies, noise 
and baseline shifts. The ST spectrogram displayed in Fig.7.b shows that in the frequency band $[5 \mathrm{~Hz}-22.5 \mathrm{~Hz}]$, the energy belongs essentially to the QRS complexes where the energy corresponding to the artifacts and baseline wander can be seen below $5 \mathrm{~Hz}$. This allows us to isolate these QRSs in the time-frequency domain. Fig.7.c illustrates Shannon energy computed using the selected time-frequency matrix.

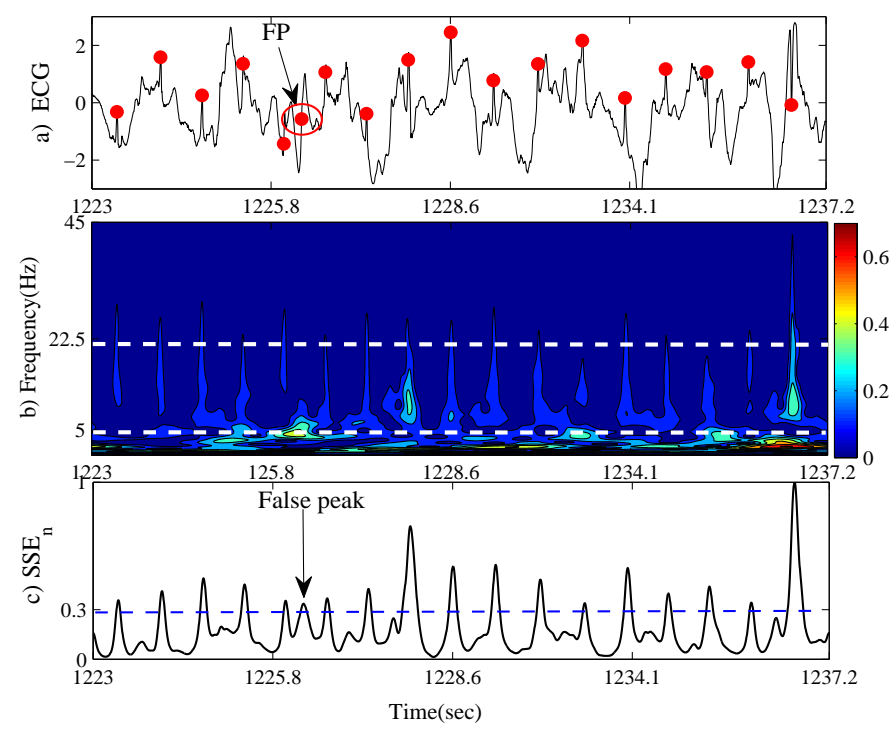

Figure 7: a) Plot of ECG waveform, segment of record 105: [1223s - 1237.2s] with different artifacts; b) ST spectrogram of the ECG, the color bar scale shows the relationship between spectrogram amplitude and color; c) Shannon energy of the selected frequency band. FP indicates a false detection. The bullets represent the position of detected $\mathrm{R}$ peaks.

Record 108 is with a borderline indication of a first degree of auriculoventricular blocks, fusion PVC and severe morphological changes. The ECG waveform given in Fig.8.a is taken from the noisy episode of the record [25]. It displays peaked $\mathrm{P}$ waves quite uncharacteristic in morphology of typical 
$\mathrm{P}$ waves. This record also exhibits muscle noise and considerable baseline shifts. In the spectrogram (Fig.8.b), one can see that although their small amplitude, the QRS complexes are clearly isolated from the rest of the signal components. The peaked $\mathrm{P}$ waves show a frequency content slightly higher than $5 \mathrm{~Hz}$. Such waves could be detected as QRS complexes because of their high slopes. However, the high temporal resolution of the S-Transform allowed us to accurately locate all $\mathrm{R}$ peaks see (Fig.8.c).

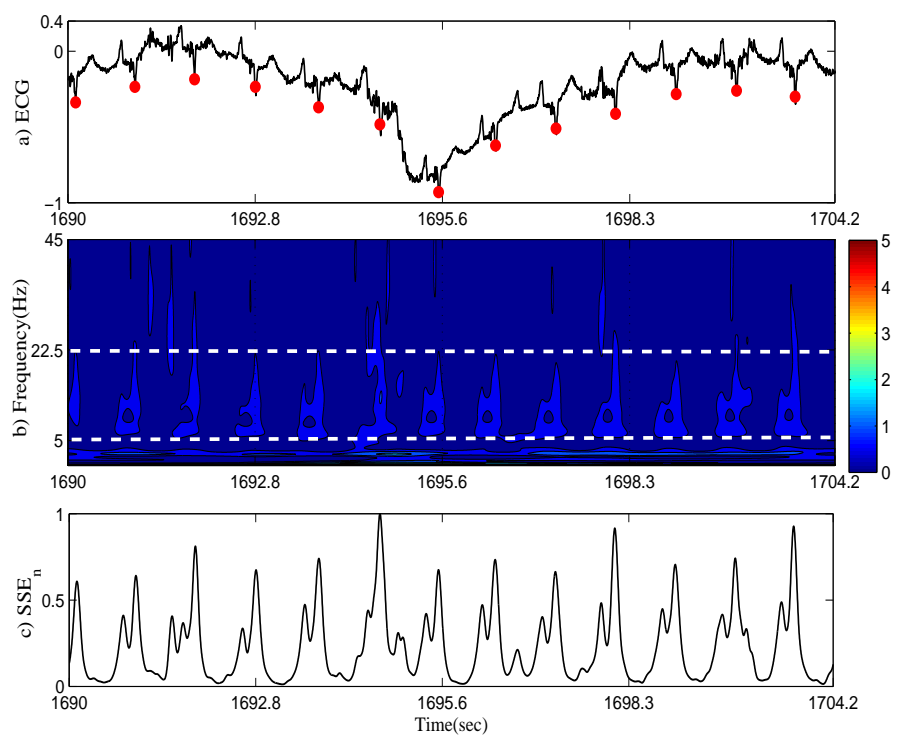

Figure 8: a) Plot of ECG waveform, segment of record 108: [1690s - 1704.2s]; b) ST spectrogram of the ECG, the color bar scale shows the relationship between spectrogram amplitude and color; c) Shannon energy of the selected frequency band. The bullets represent the position of detected $\mathrm{R}$ peaks.

In record 203 (a man of 43 years old), the PVCs are multiform. There are many QRS morphologies, sudden $\mathrm{R}$ amplitude variation and heart rate changes. There is considerable noise including muscle artifact and baseline 
shifts. This is a very difficult record, even for humans [25]. Fig.9.a displays an original segment among the most challenging of this record. Heartbeats of a smaller amplitude are clearly separated from the other components in the time-frequency image (Fig.9.b). Therefore, all R peaks are correctly located (see Fig.9.c).
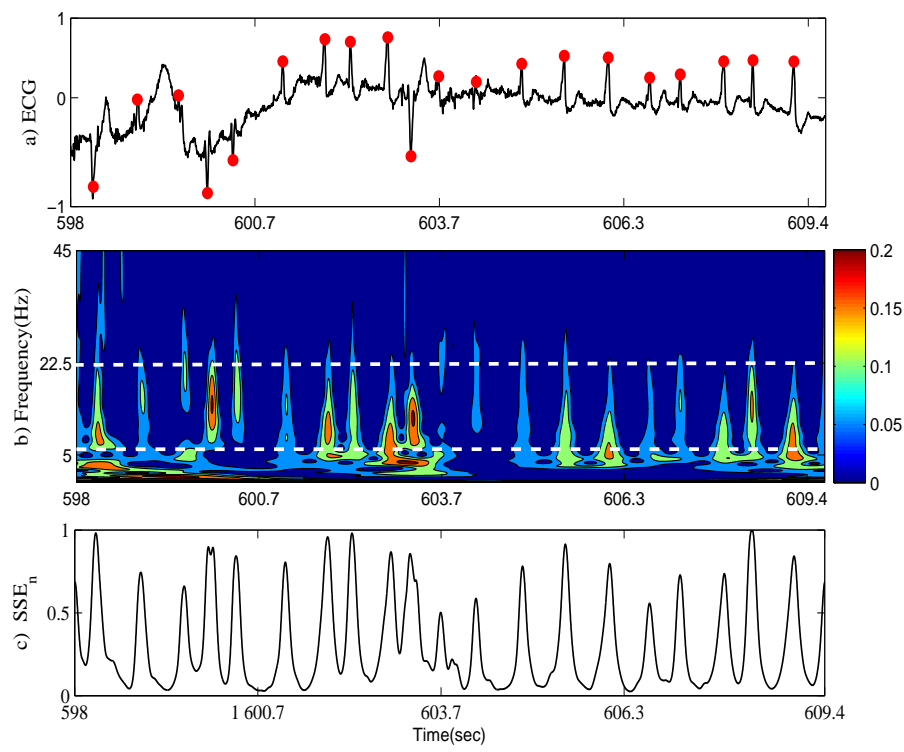

Figure 9: a) Plot of ECG waveform, segment of record 203: [598s-609.4s]; b) ST spectrogram of the ECG, the color bar scale shows the relationship between spectrogram amplitude and color; c) Shannon energy of the selected frequency band. The bullets represent the position of detected $R$ peaks.

In Fig.10.a, the selected segment of record 228 (a woman of 80 years old) shows multiform PVCs, small $\mathrm{R}$ waves with considerable baseline drift. The detection parameters illustrate clearly the localization of all $\mathrm{R}$ peaks (see Fig.10. and c).

The presented results showed that the proposed method is precise in 


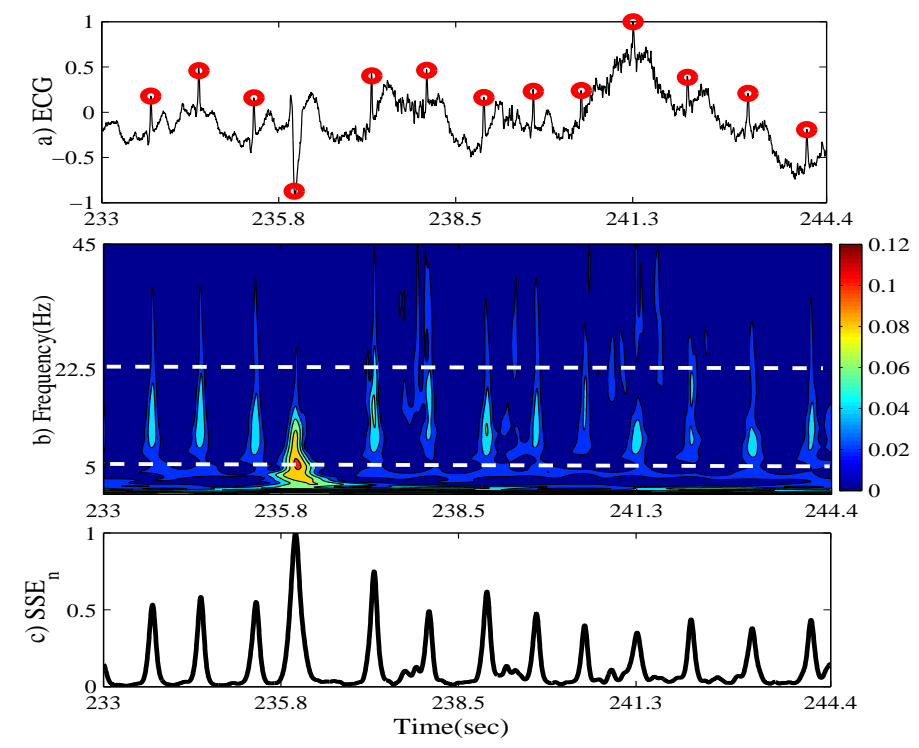

Figure 10: a) Plot of ECG waveform, segment of record 228: [233.3s-244.7s] with muscle artifacts and baseline drift; b)ST spectrogram of the ECG, the color bar scale shows the relationship between spectrogram amplitude and color; c)Shannon energy of the selected frequency band. The bullets represent the position of detected $R$ peaks.

presence of high frequency noises as shown for the case of record 104 (Fig.6), multi patterns problems signals such as the record 203 (Fig.9), broad variety of QRS morphologies and baseline shift as noticed when screening the segments of the record 105 (Fig.7). We note also that the $\mathrm{R}$ peaks beats are detected using the initial threshold $\lambda=0.3 \max \left(S S E_{n}\right)$.

Based on the frequency characteristics of the QRS complex, it might appear that a bandpass filtering might be used to localize the QRS complex. However, the traditional filters when applied to non-stationary signals such as QRS complex, might introduce jitter to the fiducial points (R-wave). Hence, the RR intervals might have high uncertainty. However, the time-frequency 
approach used here is devoid of this problem and the proposed approach reliably localizes the $\mathrm{R}$ waves.

Moreover, statistical parameters are used in order to compare detection algorithms. The sensitivity $S_{e}$, the positive predictivity $P_{+}$and the error rate $E_{r}$ are defined respectively as:

- $S_{e}=\frac{T P}{T P+F N}$

- $P_{+}=\frac{T P}{T P+F P}$

- $E_{r}=\frac{F N+F P}{T B}$

where $T P$ are the correctly detected beats (True Positive), FP are falsely detected beats (False Positive), FN are the undetected beats (False Negative) and $T B$ the total analyzed beats. The sensitivity is used for evaluating the ability of the algorithm to detect true beats, the positive predictivity is used for evaluating the ability of the algorithm to discriminate between true and false beats and the error rate is used for evaluating the accuracy of the algorithm. In the present application, the $\mathrm{R}$ peaks are detected exactly or very closely to the annotated times. When a peak is shifted with respect to the correct position, this shift is less than $27.8 \mathrm{~ms}$ (10 samples). The decision-making rule is that a detection is true when the $\mathrm{R}$ peak is detected within $50 \mathrm{~ms}$ from the annotated time, otherwise the detection is false. The interval of $50 \mathrm{~ms}$ is mentioned as a wider margin. It is lower than the margin of $100 \mathrm{~ms}$ mentioned in [22] and $75 \mathrm{~ms}$ mentioned in [9].

The achieved results reported in table 1 show that our algorithm succeeded in detecting correctly $99.84 \%$ of total beats, further, the positive predictivity $P_{+}=99.91 \%$ and the error rate $E r=0.25 \%$. Some records (105, 
108, and 201) leading to an error rate higher than $1.2 \%$ suffer from changes in signal quality, baseline shifts, artifacts and QRS waves with highly unusual morphologies. 
On the other hand, we performed a comparison with several other approaches cited in literature, classical algorithm [2], Digital filter based method [5], wavelet based methods [15], a zero crossing method [9], combined adaptive thresholds [10] and Combining algorithms [22] (see table 2).

\section{Conclusion}

In this paper, a new $\mathrm{R}$ peak detection algorithm based on S-Transform and Shannon energy is proposed and tested with respect to the MIT-BIH arrhythmia database. To our knowledge, it is the first time that the STransform has been applied to QRS detection.

In this work, we pointed out the advantage of using S-Transform to access to the frequency content of the QRS complexes without recourse to any preprocessing stage and the advantage of using Shannon energy to highlight all beats, even those having a small amplitude.

The performance of our algorithm is evaluated for all 48 records of the database. The statistical indices are higher than, or comparable to those, published in the literature. Moreover, to be more convincing, we have illustrated the possibility of detecting $R$ peak positions on some selected ECG segments with complicated patterns.

Further improvements to the algorithm may be easily achieved by extracting more features from the ST-matrix for other ECG signal processing tasks such as arrhythmia classification.

Finally, we hope that the proposed algorithm can be tested or by an independent expert excluding possible author's influence. 


\section{References}

[1] B.U. Köhler, C. Hennig, R. Orglmeister, The principles of software QRS detection, IEEE Eng. Med. Biol. Mag. 21(3)(2002) 42-57.

[2] J. Pan, W. J. Tompkins, A real-time QRS detection algorithm, IEEE Trans. Biomed. ENG. 32(3)(1985) 230-236.

[3] P.S.Hamilton, W. J. Tompkins, Quantitative Investigation of QRS Detection Rule Using the MIT/BIH Arrhythmia Database, IEEE Trans. Biomed. ENG. 33(12)(1986) 1157-1165.

[4] N. Arzeno, Zhi-De-Deng, C. Poon, Analysis of First-Derivative Based QRS Detection Algorithms, IEEE Trans. Biomed. ENG. 55(2)(2008) 478-484.

[5] V.X. Afonso, W.J.Tompkins, T.Q. Nguyen,S. Luo, ECG Beat Detection Using Filter Banks, IEEE Trans. Biomed. ENG. 46(2)(1999) 192-202.

[6] M. Okada, A digital filter for the QRS complex detection, IEEE Trans. Biomed. ENG. 26(12),(1999) 700-703.

[7] S.Tabakov, M.Lliev, V.Krasteva, Online digital filter and QRS detector applicable in low resource ECG monitoring systems, Annals of Biomedical Engineering. 36(11)(2008) 1805-1815.

[8] D. Benitez, P.A. Gaydecki, A.Zaidi, A.P.Fitzpatrick, The use of the Hilbert transform in ECG signal analysis, Computers in Biology and Medicine. 31(5)(2001) 399-406. 
[9] B.U.Köhler, C. Hennig, R. Orglmeister, QRS Detection Using Zero Crossing Counts, Progress in Biomedical Research. 8(3)(2003) 138-145.

[10] I. I. Christov, Real time electrocardiogram QRS detection using combined adaptive threshold, Biomedical Engineering Online, 3(28)(2004) $1-9$

[11] C.Li, C.Zheng, C.Tai, Detection of ECG characteristic points using wavelet transforms, IEEE Trans. Biomed. ENG. 42(4)(1995) 21-28.

[12] B. Abibullaev, H.Don.Seo, A new QRS detection method using wavelets and Artificial Neural-Networks, Springer J. Med. Syst. 35(2011) 683-691.

[13] G.Ruchita, A.K.Sharma, Detection of QRS complexs of ECG recording based on wavelet transform using Matlab, Int. J. Eng. SCI. 2(7)(2010) 3038-3034.

[14] A.N.Dinh, D.K.Kumar, N.D.Pah and P. Burton, Wavelet for QRS detection, Engineering in medicine and Biology society, 23rd Conference of IEEE. (2002) 7803-7211.

[15] Z. Zidelmal, A. Amirou, M. Adnane, A Belouchrani. QRS detection using wavelet coefficients. Computer Methods and Programs in Biomedicine, (107) (3) (2012) 490-496.

[16] S.W.Chen, C.H.Chen, H.L.Chan, A real-time QRS method based on moving-averaging incorporating with wavelet denoising, Comput. Method. Program. Biomed. 82(3)(2006) 187-195. 
[17] Q. Xue, Y.H.Hu, J. Tompkins, Neural-network-based adaptive matched filtering for QRS detection, IEEE Trans. Biomed. ENG. 39(4)(1992) 317-329.

[18] S.S.Mehta, N.S.Ligayat, Comparative study of QRS detection in single lead and 12-lead ECG based on entropy and combined entropy criteria using Support Vector Machine, Journal of Theoretical and Applied Information Technology 3(2)(2007) 8-18.

[19] R Poli, S.Cagnoni, G. Valli, Genetic design of optimum linear and nonlinear QRS detectors, IEEE Trans. Biomed. ENG. 42(11)(1995) $1137-1141$.

[20] D.A.Coast, R.M.Stern,G.G.Cano, S.A.Briller, An approach to cardiac arrhythmia analysis using hidden Markov models, IEEE Trans. Biomed. Eng. 37(9)(1990) 826-836.

[21] I. Saini, D. Singh, A. Khosla, QRS detection using K-Nearest Neighborr algorithm $(\mathrm{KNN})$ and evaluation on standard ECG databases, Journal of Advanced Research 4(4)(2013) 331344.

[22] C. Meyer, J.F.Gavela, M.Harris, Combining algorithms in automatic detection of QRS complexes in ECG signals, IEEE Trans. Inf. Technol. B. 10(3)(2006) 468-475.

[23] J.C.T.B. Moraes, M. Seixas, F.N.Vilani, E.V. Costa, A QRS complex detection algorithm using electrocardiogram leads, Comp.in.Card. 29(2002) 205-208. 
[24] R.G. Stockwell, L. Mansinha and R.P.Lowe Localisation of the complex spectrum: The S-Transform. IEEE Trans. signal processing, 44 (4), (1996), 998-1001.

[25] R.Mark, G.Moody, MIT-BIH arrhythmia database, http://www.physionet.org/physiobank/database/mitdb/, (accessed:26 September 2013).

[26] R.G. Stockwell Why use the S-Transform ?. AMS Pseudodifferential operators: partial differential equations and timefrequency analysis, 52, (2007), 279-309.

[27] A. Moukadem, A.Dieterlen, N.Hueber and C.Brandt A robust heart sounds segmentation module based on S-Transform. Biomedical signal processing and control, 8(13),2013, 273-281. 
Table 1: The experimental results

\begin{tabular}{|c|c|c|c|c|c|c|c|}
\hline Rec & TB & $\mathrm{TP}$ & $\mathrm{FP}$ & $\mathrm{FN}$ & $S_{e}(\%)$ & $P_{+}(\%)$ & $\operatorname{Er}(\%)$ \\
\hline 100 & 2273 & 2273 & 0 & 0 & 100.00 & 100.00 & 0.00 \\
\hline 101 & 1865 & 1864 & 2 & 1 & 99.95 & 99.89 & 0.16 \\
\hline 102 & 2187 & 2185 & 0 & 2 & 99.91 & 100.00 & 0.09 \\
\hline $10 \overline{3}$ & 2084 & 2084 & 0 & 0 & 100.00 & 100.00 & 0.00 \\
\hline 104 & 2229 & 2220 & 5 & 9 & 99.60 & 99.78 & 0.63 \\
\hline 105 & 2572 & 2550 & 10 & 22 & 99.14 & 99.61 & 1.24 \\
\hline 106 & 2027 & 2027 & 8 & 0 & 100.00 & 99.61 & 0.39 \\
\hline 107 & 2137 & 2137 & 2 & 0 & 100.00 & 99.91 & 0.09 \\
\hline 108 & 1763 & 1740 & 20 & 23 & 98.70 & 98.86 & 2.44 \\
\hline 109 & 2532 & 2532 & 0 & 0 & 100.00 & 100.00 & 0.00 \\
\hline 111 & 2124 & 2122 & 0 & 2 & 99.91 & 100.00 & 0.09 \\
\hline 112 & 2539 & 2537 & 0 & 2 & 99.92 & 100.00 & 0.08 \\
\hline 113 & 1795 & 1793 & 1 & 2 & 99.89 & 99.94 & 0.17 \\
\hline 114 & 1879 & 1877 & 1 & 2 & 99.89 & 99.95 & 0.16 \\
\hline 115 & 1953 & 1953 & 0 & 0 & 100.00 & 100.00 & 0.00 \\
\hline 116 & 2412 & 2409 & 2 & 3 & 99.88 & 99.92 & 0.21 \\
\hline 117 & 1535 & 1535 & 0 & 0 & 100.00 & 100.00 & 0.00 \\
\hline 118 & 2278 & 2273 & 0 & 5 & 99.78 & 100.00 & 0.22 \\
\hline 119 & 1987 & 1987 & 0 & 0 & 100.00 & 100.00 & 0.00 \\
\hline 121 & 1863 & 1861 & 1 & 2 & 99.89 & 99.95 & 0.16 \\
\hline 122 & 2476 & 2476 & 0 & 0 & 100.00 & 100.00 & 0.00 \\
\hline 123 & 1518 & 1518 & 0 & 0 & 100.00 & 100.00 & 0.00 \\
\hline 124 & 1619 & 1619 & 1 & 0 & 100.00 & 99.94 & 0.06 \\
\hline 200 & 2601 & 2597 & 2 & 4 & 99.85 & 99.92 & 0.23 \\
\hline 201 & 1963 & 1938 & $\overline{4}$ & 25 & 98.73 & 99.79 & 1.48 \\
\hline 202 & 2136 & 2135 & 1 & 1 & 99.95 & 99.95 & 0.09 \\
\hline 203 & 2980 & 2969 & 9 & 11 & 99.63 & 99.70 & 0.67 \\
\hline 205 & 2656 & 2653 & 0 & 3 & 99.89 & 100.00 & 0.11 \\
\hline 207 & 1860 & 1847 & 8 & 13 & 99.30 & 99.57 & 1.13 \\
\hline 208 & 2955 & 2952 & 2 & 3 & 99.90 & 99.93 & 0.17 \\
\hline 209 & 3005 & 3004 & $\overline{1}$ & 1 & 99.97 & 99.97 & 0.07 \\
\hline 210 & 2650 & 2647 & 2 & 3 & 99.89 & 99.92 & 0.19 \\
\hline 212 & 2748 & 2748 & 0 & 0 & 100.00 & 100.00 & 0.00 \\
\hline 213 & 3251 & 3250 & 0 & 1 & 99.97 & 100.00 & 0.03 \\
\hline 214 & 2262 & 2257 & 2 & 5 & 99.78 & 99.91 & 0.31 \\
\hline 215 & 2363 & 2363 & 1 & 0 & 100.00 & 99.96 & 0.04 \\
\hline 217 & 2208 & 2205 & 2 & 3 & 99.86 & 99.91 & 0.23 \\
\hline 219 & 2154 & 2154 & 0 & 0 & 100.00 & 100.00 & 0.00 \\
\hline 220 & 2048 & 2048 & 1 & 0 & 100.00 & 99.95 & 0.05 \\
\hline 221 & 2427 & 2424 & 1 & 3 & 99.88 & 99.96 & 0.16 \\
\hline 222 & 2483 & 2483 & 0 & 0 & 100.00 & 100.00 & 0.00 \\
\hline 223 & 2605 & 2604 & 0 & 1 & 99.96 & 100.00 & 0.04 \\
\hline 228 & 2053 & 2046 & 4 & 7 & 99.66 & 99.80 & 0.54 \\
\hline 230 & 2256 & 2255 & 3 & 1 & 99.96 & 99.87 & 0.18 \\
\hline 231 & 1571 & 1571 & 0 & 0 & 100.00 & 100.00 & 0.00 \\
\hline 232 & 1780 & 1779 & 0 & 1 & 99.94 & 100.00 & 0.06 \\
\hline 233 & 3079 & 3073 & 0 & 6 & 99.81 & 100.00 & 0.19 \\
\hline 234 & 2753 & 2749 & 1 & 4 & 99.85 & 99.96 & 0.18 \\
\hline All & 108494 & 108323 & 97 & 171 & 99.84 & 99.91 & 0.25 \\
\hline
\end{tabular}


Table 2: Comparison with other algorithms

\begin{tabular}{llll}
\hline Method & $S_{e}(\%)$ & $P_{+}(\%)$ & $\operatorname{Er}(\%)$ \\
\hline The proposed method & 99.84 & 99.91 & 0.25 \\
J.Pan et al.. (1985) & 99.75 & 99.54 & 0.71 \\
X.Valtino et al. (1999) & 99.59 & 99.56 & -- \\
Köhler et al.(2003) & 99.70 & 99.57 & -- \\
I.I.Christov (2004) & 99.74 & 99.65 & -- \\
C. Meyer et al. $(2006)$ & 99.47 & 99.54 & 0.98 \\
Z.Zidelmal et al. (2012) & 99.64 & 99.82 & 0.54 \\
\hline
\end{tabular}

